\title{
Falamos da Mesma Criança? Concordância Mãe - Pai - Professores na Avaliação do Temperamento de Crianças Portuguesas
}

\author{
Is that the Same Child? Parents' and Teachers' Agreement in Temperament \\ Assessment of Portuguese Children
}

\author{
Maria João Seabra-Santos ${ }^{*}{ }^{a} \&$ Margarida Sousa Almeida ${ }^{b}$ \\ ${ }^{a}$ Universidade de Coimbra, Coimbra, Distrito de Coimbra, Portugal \\ \& ${ }^{b}$ Hospital Pediátrico de Coimbra, Coimbra, Distrito de Coimbra, Portugal
}

\begin{abstract}
Resumo
Com este trabalho pretendeu-se analisar o grau de concordância entre diferentes informadores mães, pais, professores do ensino regular e dos apoios educativos (quando aplicável) - na avaliação do temperamento de crianças com 2 a 7 anos de idade. O estudo incidiu sobre uma amostra de 138 crianças, distribuídas por quatro grupos com diferentes características desenvolvimentais: síndrome de Down, Autismo, Nascimento Prematuro e Desenvolvimento Normal. Os informadores preencheram a Temperament Assessment Battery for Children - Revised nas versões para pais ou para professores. Encontraram-se correlações elevadas entre informadores que observam a criança no mesmo contexto e baixas entre avaliações de adultos pertencentes a contextos diferentes. Os resultados são discutidos em função das suas possíveis implicações para a avaliação do temperamento de crianças.

Palavras-chave: Concordância inter-avaliador, questionários, temperamento, pré-escolar.

Abstract

This study was intended to address the issue of convergence between informants (mother, father, regular teacher and special education teacher, whenever applicable) when assessing the temperament of children between 2 and 7 years old. The study was based on a sample of 138 children, distributed into four groups with different developmental characteristics: Down syndrome, Autism, Premature Birth, and Normal Development. Informants filled in the Temperament Assessment Battery for Children - Revised in its parents' or teachers' versions. Whereas correlations between assessments made by adults of different contexts were low, high correlations were found between informants who observe the child in the same context. Results are discussed in terms of their possible implications for the assessment of children's temperament.

Keywords: Inter-rater agreement, rating scales, temperament, preschooler.
\end{abstract}

O recurso a várias fontes de informação e a informadores diversos constitui um paradigma da avaliação psicológica de crianças (Major, 2011; Seabra-Santos, 2000). Subjacente está a ideia de que o comportamento é variável em função dos contextos nos quais a criança interage e das pessoas e condições presentes em cada um deles. Por conseguinte, para obter uma imagem minimamente credível e representativa da criança torna-se necessário recolher amostras do seu comportamento em vários contextos, o que se consegue recorrendo a diferentes informadores. Porém, a coleta de dados a partir de vários informadores, se por um lado favorece a obtenção de uma imagem mais

\footnotetext{
"Endereço para correspondência: Faculdade de Psicologia e de Ciências da Educação, Universidade de Coimbra, Rua do Colégio Novo, Apartado, 6153, Coimbra Portugal 3001-802. E-mail: seabramj@fpce.uc.pt.

Agradecimentos: As autoras agradecem a todos os pais e professores que colaboraram no presente trabalho através do preenchimento de questionários.
}

completa e integrada do sujeito em avaliação, por outro lado coloca a questão da concordância/discordância entre eles. O presente artigo pretende analisar o grau de concordância entre diferentes informadores na avaliação do temperamento de crianças em idade pré-escolar e início da idade escolar.

Embora esta questão tenha sido bastante explorada no que diz respeito à avaliação do comportamento da criança (Berg-Nielsen, Solheim, Belsky, \& Wichstrom, 2012; Major, 2011; Miner \& Clarke-Stewart, 2008), o mesmo não se passa em relação ao seu temperamento. O construto "temperamento", definido como o conjunto de aspetos estilísticos ou o "como" do comportamento (Carey \& McDevitt, 2012; Chess \& Thomas, 1999) é, ele próprio, pouco consensual, existindo diferentes modelos teóricos suscetíveis de o enquadrar. Autores representativos, neste domínio, são Alexander Thomas e Stella Chess, Arnold Buss e Robert Plomin, Hill Goldsmith, Jerome Kagan, Mary Rothbart, Sam Putnam e William Carey, 
para referir somente alguns dos que mais contribuíram para o avanço da reflexão teórica e da investigação sobre o temperamento. Porém, há elementos na caraterização deste conceito em relação aos quais a unanimidade é clara, nomeadamente quanto ao seu surgimento precoce, o facto de se refletir em diferenças individuais no comportamento e na personalidade, o seu substrato neurobiológico e a sua relativa estabilidade, embora podendo ser influenciado por condições ambientais (Goldsmith et al., 1987).

Pais e professores são os informadores a quem mais se recorre em processos de avaliação psicológica de crianças, na medida em que eles são os adultos em melhores condições para apreciar o comportamento e desenvolvimento da criança. Na verdade, os pais têm possibilidade de observar os seus filhos num grande número de situações, incluindo aquelas que ocorrem com pouca frequência, e ao longo de períodos alargados de tempo, pelo que possuem uma perspetiva temporal que lhes permitirá compreender qual o comportamento habitual da criança (Rothbart \& Bates, 2006). Deste modo, os pais detêm uma posição relativamente aos seus filhos que lhes permite fornecer uma grande quantidade de informações relevantes sobre eles (Kristal, 2005; McClowry, Halverson, \& Sanson, 2003; Rochette, 2008; Rothbart \& Bates, 2006). Por seu turno os professores poderão ter uma ideia mais realista da situação, dado que possuem uma base normativa para apreciar o comportamento da criança, que lhes advém do facto de lidarem diariamente com um grande número de alunos e de poderem observá-los num ambiente mais estruturado que o meio familiar (Berg-Nielsen et al., 2012; Major, 2011).

Para avaliar as caraterísticas socioemocionais de crianças, os inventários respondidos por adultos constituem o método mais frequentemente utilizado, em particular quando se trata de crianças em idade pré-escolar, uma vez que o nível de desenvolvimento ainda incipiente desta população nos planos verbal, cognitivo e grafomotor diminuem consideravelmente ou excluem, mesmo, a possibilidade de utilizar a maioria das técnicas usadas na avaliação de crianças mais velhas ou de adultos, tais como os inventários de autorresposta, as entrevistas ou algumas técnicas projetivas (Keith \& Campbell, 2004). Em comparação com outros métodos comportam a vantagem óbvia de recolherem amostras de comportamento em situações variadas e por períodos longos (Kagan \& Fox, 2006). Para além disso, representam um modo de avaliação económico, uma vez que não consomem nem muito tempo, nem exigem grande preparação por parte dos profissionais que os administram (Kristal, 2005; Rothbart \& Bates, 2006). São, igualmente, fáceis de analisar e descrevem a criança em vários domínios (Keith \& Campbell, 2004), podendo dar lugar à elaboração de perfis individuais. Paralelamente, os questionários podem apresentar também alguns aspetos negativos relacionados com as suas fracas qualidades métricas (aspeto este que é mais saliente nos instrumentos destinados à idade pré-escolar; Keith \& Campbell, 2004; Seabra-Santos, 2000), e com a falta de concordância entre avaliações efetuadas por diferentes informadores (Martin, 2010; Rothbart \& Bates, 2006).
Várias razões podem ser invocadas para explicar a discordância entre informadores. Por um lado, se os diferentes adultos lidam com a criança em situações distintas, por exemplo, em casa e na escola, será de esperar um certo grau de discordância em relação ao comportamento observado e às caraterísticas da criança que tal comportamento permite inferir (Keith \& Campbell, 2004; Rochette, 2008). A própria presença de pessoas diversas na situação pode induzir na criança a manifestação de comportamentos diferentes, aspeto este particularmente presente nas crianças pré-escolares, dado que estas são altamente maleáveis à ação do adulto e vulneráveis aos aspetos particulares de cada situação. Por conseguinte, a discordância entre informadores não resulta necessariamente de qualquer imprecisão no processo de avaliação, mas antes pode traduzir diferenças reais no comportamento observado.

Acresce, por outro lado, que quando o comportamento e as caraterísticas da criança são reportados por terceiros (em vez de observados diretamente por observadores treinados), os relatos dos informadores serão filtrados pelo conjunto de significados que lhes atribuirão, refletindo a relação entre os dois, tal como é vista pelo adulto (Martin, 2010). Tal circunstância introduz neste processo uma margem incontornável de subjetividade (Rochette, 2008; Rothbart \& Bates, 2006). Por sua vez, as perceções podem ser influenciadas por variáveis de natureza cultural (Weisz, Sigman, Weiss, \& Mosk, 1993), ou pessoal, nomeadamente traços de personalidade (Hayden, Durbin, Klein, \& Olino, 2010; Kroes, Veerman, \& De Bruyn, 2005), a presença de depressão, ansiedade ou outro tipo de psicopatologia no informador (Berg-Nielsen et al., 2012; Carey \& McDevitt, 2012; Macedo et al., 2011) ou, ainda, por diferentes expectativas e uma focalização diferenciada da atenção dos observadores em aspetos particulares do comportamento (Klyce, Conger, Conger, \& Dumas, 2011). Nestes casos a discordância não estará relacionada com uma intenção deliberada de falsear as informações prestadas mas, antes, refletirá o modo como cada pessoa genuinamente analisa e descreve aquilo que observa.

O maior ou menor grau de conhecimento da criança pode, também, influenciar a concordância entre informadores. No caso dos professores demonstrou-se que a concordância é superior quando ambos conhecem a criança há vários meses e têm muita experiência com crianças da mesma faixa etária (Anthony, Anthony, Morrel, \& Acosta, 2005). Neste mesmo sentido, as descrições das mães tendem a ser mais precisas quando elas têm vários filhos do que quando são primíparas (Forman et al., 2003). O facto de permanecerem menos tempo com a criança poderá ser responsável pelos níveis mais baixo de concordância com os professores obtidos pelos pais (homens), em comparação com as mães (Bishop, Spence, \& McDonald, 2003). $\mathrm{O}$ grau de concordância entre os progenitores quanto à presença de um problema de comportamento na criança é também superior em casais com níveis mais elevados de satisfação conjugal, que percecionam os seus cônjuges como apoiantes e que partilham o envolvimento nos cuidados prestados à criança (Goldberg, 1990). 
Por outro lado, há comportamentos e caraterísticas da criança que suscitam um maior grau de concordância do que outros. Assim, a concordância é superior: (a) relativamente a amostras de crianças sinalizadas (Keogh \& Bernheimer, 1998) ou com caraterísticas mais extremas por exemplo, quanto à inibição (Bishop et al., 2003), em comparação com amostras de crianças normais; (b) para comportamentos de tipo externalizante do que para os de tipo internalizante (Achenbach, McConaughy, \& Howell, 1987; Duhig, Renk, Epstein, \& Phares, 2000; Keogh \& Bernheimer, 1998; Merrell, 2002); (c) em relação a problemas de comportamento do que a aptidões sociais (Merrell, 2002); (d) relativamente a caraterísticas temperamentais que são mais salientes e importantes para o ajustamento e desempenho escolar tais como, por exemplo, a adaptabilidade e a persistência (Field \& Greenberg, 1982).

A avaliação do temperamento das crianças tem merecido pouca atenção por parte da investigação, no que toca à questão da concordância entre informadores. Os poucos estudos que abordam este tópico encontram correlações baixas entre as classificações atribuídas por informadores pertencentes a contextos diferentes: por exemplo, coeficientes oscilando entre 0,22 e 0,47 entre as respostas de mães e de professores à Temperament Assessment Battery for Children - Revised (TABC-R; Martin \& Bridger, 1999). Os coeficientes calculados entre resultados de informadores pertencentes ao mesmo contexto atingem valores moderados: por exemplo, quando avaliada a concordância entre mãe e pai os coeficientes de correlação variaram entre 0,34 e 0,66 no estudo de Martin e Bridger (1999), e entre 0,51 e 0,68 numa investigação sobre o School-Age Temperament Inventory (McClowry, 1995, citado por McClowry et al., 2003). A concordância é superior relativamente a características mais observáveis do que quando se reporta a outras mais "cobertas". Assim, utilizando a TABC-R, Martins (2010) obtém níveis de concordância baixos entre pais e professores no que toca à dimensão Inibição $(0,16)$ e moderados relativamente à dimensão Impulsividade $(0,48)$. Também Muris e Meesters
(2009) comparando as classificações atribuídas pelos pais com as do próprio nas várias escalas do Early Adolescent Temperament Questionnaire - Revised (EATQ-R) chegam a coeficientes de correlação que variam entre 0,33 para a afiliação, frustração e sensibilidade percetiva, e 0,59 para o nível de atividade.

Sendo os questionários respondidos por pais e por professores os instrumentos mais usados nas investigações conduzidas na área do temperamento (Martin, 2011), a análise feita no presente estudo sobre a concordância entre informadores na avaliação do temperamento de crianças pré-escolares, recorrendo a quatro informadores diferentes e em amostras com características diversas, afigura-se-nos relevante. Os dados são analisados recorrendo a dois métodos: 1) coeficientes de correlação entre as pontuações atribuídas pelos vários informadores; e 2) comparações entre médias. Com base na escassa literatura disponível, esperamos encontrar coeficientes de correlação mais elevados para a característica "impulsividade" do que para a "inibição", e mais elevados entre informadores que lidam com a criança num mesmo contexto do que para informadores que lidam com ela em contextos diferentes. Finalmente, pretendemos esclarecer o grau em que as diferenças entre amostras, do ponto de vista da presença de patologias específicas, afeta o nível de concordância entre os informadores.

\section{Método}

\section{Participantes}

O estudo incidiu sobre uma amostra de 138 crianças de nacionalidade portuguesa com idades compreendidas entre os 2 e os 7 anos, 36 das quais com Síndrome de Down, 33 com Autismo, 33 Nascidas Prematuramente (subamostras clínicas) e 36 crianças com Desenvolvimento Normal, sem fatores de risco conhecidos e, por consequência, sem quaisquer tipos de apoios educativos a nível escolar (Tabela 1). As crianças das subamostras clínicas possuíam diagnósticos formais, realizados por equipas multidisciplinares no âmbito de consultas especializadas.

Tabela 1

Caracterização da Amostra

\begin{tabular}{lcccc}
\hline & Sínd. Down & Autismo & Prematuros & Des. Normal \\
\hline Número de casos & 36 & 33 & 33 & 36 \\
Idade média em meses $(D P)$ & $58,3(16,9)$ & $65,6(15,3)$ & $55,8(17,5)$ & $57,5(16,6)$ \\
Sexo & & & & \\
$\quad$ Feminino & 14 & 6 & 11 & 14 \\
$\quad$ Masculino & 22 & 27 & 22 & 22 \\
Nível Socioeconómico & 17 & 12 & 21 & 19 \\
$\quad$ Baixo & 15 & 17 & 8 & 13 \\
$\quad$ Médio & 4 & 3 & 4 & 4 \\
$\quad$ Elevado & $9,9(5,3)$ & $11,2(4,4)$ & $10,9(4,4)$ & $11,6(4,5)$ \\
Média anos escol. mãe $(D P)$ & &
\end{tabular}


Seabra-Santos, M. J. \& Almeida, M. S. (2014). Falamos da Mesma Criança? Concordância Mãe - Pai - Professores na Avaliação do Temperamento de Crianças Portuguesas.

A relação entre o número de rapazes e o de raparigas é de aproximadamente 2 para $1 \mathrm{em}$ todas as subamostras exceto na composta por crianças com Autismo, em que essa relação é de cerca de 4 para 1 . A seleção das crianças com Desenvolvimento Normal teve em conta as variáveis idade, sexo e nível socioeconómico, de forma a constituir uma subamostra com características idênticas às das subamostras clínicas. O nível socioeconómico das famílias, estimado com base na profissão dos pais, é maioritariamente baixo (trabalhadores não especializados da indústria e da construção civil, empregados de balcão no pequeno comércio, etc.) ou médio (técnicos intermédios independentes, empregados de escritório, enfermeiros, professores do ensino primário e secundário, etc.). A idade média das mães desta amostra é de cerca de 36 anos $(D P=6,17)$ e a dos pais é de cerca de 38 anos $(D P=6,48)$, sendo o seu número médio de anos de escolaridade igual a 10,86 $(D P=4,68)$ e a $9,70(D P=4,69)$, respetivamente. Todos os professores dispõem de formação específica média ou superior em educação.

\section{Instrumento}

No presente estudo foi utilizada a Bateria de Avaliação do Temperamento Infantil - Forma Revista (Temperament Assessment Battery for Children - Revised, TABC-R, Martin \& Bridger, 1999), preenchida por pais e por professores de crianças com idades compreendidas entre os 2 e os 7 anos. Esta bateria baseia-se na teoria neuropsicológica de Jeffrey Gray, que destaca a presença de dois grandes sistemas neurológicos: um Sistema de Inibição Comportamental e um Sistema de Ativação Comportamental (Gray, 1972, 1985, 1987, 1991, citado por Martin \& Bridger, 1999). Assim, a TABC-R avalia o temperamento infantil por meio de duas escalas: uma de Inibição - que mede a tendência da criança para evitar situações sociais novas, hesitar em se aproximar de estranhos e ser cautelosa quando se envolve em tarefas novas; e uma escala de Impulsividade, composta por três subescalas, que avaliam, respetivamente, a dificuldade em controlar as emoções, reagindo às frustrações com comportamentos como gritar ou chorar (Emotividade Negativa), a dificuldade em controlar comportamentos motores em situações que o requeiram (Nível de Atividade) e a dificuldade em manter a atenção durante períodos prolongados ou em aprender tarefas novas que envolvam alguma dificuldade (Falta de Persistência em Tarefa).

A TABC-R possui duas formas, uma destinada aos pais, composta por 37 itens, e outra aos professores/ educadores de infância, composta por 29 itens, traduzindo características da criança observáveis em casa e na escola, respetivamente. Embora as duas formas não sejam exatamente iguais, porquanto na análise fatorial da versão para professores o Nível de Atividade e a Falta de Persistência em Tarefa saturam num mesmo fator, os autores recomendam a derivação do mesmo conjunto de resultados para as duas versões, no sentido de proporcionar uma maior comparabilidade entre os dados recolhidos a partir dos contextos casa e escola (Martin \& Bridger,
1999). Trata-se, pois, de duas formas paralelas de um mesmo instrumento, com alguns itens comuns e outros a remeterem para situações de natureza familiar ou escolar, consoante a versão considerada. Os itens são apresentados em termos de frequência da ocorrência do comportamento em causa numa escala de 7 pontos, em que (1) representa "quase nunca" e (7) "quase sempre". Os resultados são calculados de modo a que um valor elevado significa sempre que a característica respetiva está muito presente na criança em causa.

$\mathrm{O}$ processo de adaptação da TABC-R para português constou de tradução por especialista em avaliação psicológica com um bom domínio da língua inglesa e subsequente retroversão, revista por um dos autores da Bateria. Seguiu-se uma fase de utilização experimental, em que foi pedido a pais, professores e psicólogos que comentassem a clareza dos itens. Embora não tenham sido derivadas normas deste instrumento para a população portuguesa, estudos preliminares com amostras de crianças da população geral (Almeida \& Seabra-Santos, 2010; Martins, 2010) permitiram verificar que os resultados obtidos para as dimensões e subdimensões avaliadas pela TABC-R (médias e desvios-padrão) são muito semelhantes aos encontrados na população americana, o que justificou o uso das normas americanas na presente investigação. Por outro lado, os valores da consistência interna (alfa de Cronbach) na amostra total tomada para o presente estudo são elevados (0,81 e 0,79 na escala de Inibição, e 0,84 e 0,90 na escala de Impulsividade, respetivamente nas versões para pais e para professores).

\section{Procedimentos}

Para as crianças das subamostras Síndrome de Down e Autismo foram preenchidos inventários por quatro informadores diferentes: mãe, pai, professor do ensino regular e professor dos apoios educativos (isto é, com formação especializada na intervenção junto de alunos com necessidades educativas especiais, com diagnósticos vários). Para as subamostras de crianças Nascidas Prematuramente e com Desenvolvimento Normal foram obtidos dados a partir dos três primeiros informadores. A investigação foi previamente autorizada pelas entidades competentes em cada um dos contextos de coleta e envolveu a obtenção de consentimento informado por parte dos pais.

As três subamostras clínicas foram constituídas por crianças que frequentavam consultas especializadas num hospital pediátrico central, respetivamente, consulta de Trissomia 21, de Autismo e de Recém-Nascidos de Alto Risco. Os pais destas crianças foram contactados telefonicamente por um técnico do hospital, tendo-lhes sido explicados os objetivos do estudo e solicitado que preenchessem um questionário cada (mãe e pai) e que entregassem outros dois (ou um, consoante os casos) aos professores das crianças (ensino regular e ensino especial, quando aplicável). Posteriormente foram-lhes enviados os questionários pelo correio, acompanhados por uma folha de explicação do estudo a entregar aos professores, e por um envelope selado para devolver os materiais preen- 
chidos. A subamostra com desenvolvimento normal foi recolhida em três jardins-de-infância da zona centro de Portugal. Trata-se de uma amostra por conveniência, em que o contacto inicial ocorreu através dos professores, a quem foi solicitado que fizessem chegar os questionários aos pais, acompanhados pela explicação dos objetivos do estudo e por um envelope para a respetiva devolução. A importância de preencherem os questionários de forma independente foi realçada em todas as explicações.

A concordância entre informadores foi estudada de duas formas distintas: coeficientes de correlação de Pearson entre as classificações atribuídas pelos vários informadores; e comparação entre as médias das pontuações atribuídas pelos informadores recorrendo a One-Way ANOVA seguidas de análises post-hoc através do teste de Bonferroni. Todas as análises foram feitas com base em resultados $T$ em função da idade cronológica, derivados a partir das normas americanas.

\section{Resultados}

Os resultados relativos à concordância entre observadores, na amostra total e nas várias subamostras, para as escalas de Inibição e Impulsividade e igualmente para as subescalas desta última são apresentados na Tabela 2.

Tabela 2

Concordância entre as Classificações Atribuidas pelos Vários Informadores (coeficientes $r$ de Pearson)

\begin{tabular}{|c|c|c|c|c|c|c|}
\hline Amostras & $\begin{array}{c}\text { Mãe/ } \\
\text { Pai }\end{array}$ & $\begin{array}{c}\text { Prof.Reg./ } \\
\text { Prof.Ap. }\end{array}$ & $\begin{array}{c}\text { Mãe/ } \\
\text { Prof.Reg. }\end{array}$ & $\begin{array}{c}\text { Pai/ } \\
\text { Prof.Reg. }\end{array}$ & $\begin{array}{c}\text { Mãe/ } \\
\text { Prof.Ap. }\end{array}$ & $\begin{array}{c}\text { Pai/ } \\
\text { Prof.Ap. }\end{array}$ \\
\hline \multicolumn{7}{|l|}{ Total } \\
\hline Inibição & $0,78 * *$ & $0,57 * *$ & 0,14 & 0,10 & 0,16 & 0,08 \\
\hline Impulsividade & $0,68^{* *}$ & $0,80 * *$ & $0,30 * *$ & $0,38^{* *}$ & 0,20 & $0,32 *$ \\
\hline Emotiv. Neg. & $0,54 * *$ & $0,76^{* *}$ & $0,22 *$ & $0,38^{* *}$ & 0,16 & 0,26 \\
\hline Nível Atividade & $0,50^{* *}$ & $0,70 * *$ & 0,10 & 0,18 & 0,13 & 0,13 \\
\hline Falta Persist. & $0,60 * *$ & $0,78 * *$ & $0,43^{* *}$ & $0,36^{* *}$ & $0,40 * *$ & 0,24 \\
\hline Média $^{a}$ & $0,63 * *$ & $0,73 * *$ & $0,24 *$ & $0,29 *$ & 0,22 & 0,20 \\
\hline \multicolumn{7}{|l|}{ Síndrome de Down } \\
\hline Inibição & $0,80 * *$ & $0,62 * *$ & 0,33 & 0,16 & 0,11 & 0,17 \\
\hline Impulsividade & $0,70^{* *}$ & $0,74 * *$ & 0,34 & 0,31 & 0,28 & 0,19 \\
\hline Emotiv.Neg. & $0,47 * *$ & $0,67 * *$ & $-0,06$ & 0,12 & 0,21 & 0,09 \\
\hline Nível Atividade & $0,51 * *$ & $0,75^{* *}$ & 0,26 & 0,14 & 0,25 & 0,07 \\
\hline Falta Persist. & $0,52 * *$ & $0,81 * *$ & $0,49 * *$ & 0,09 & 0,43 & 0,01 \\
\hline Média $^{\mathrm{a}}$ & $0,62 * *$ & $0,73 * *$ & 0,27 & 0,17 & 0,26 & 0,13 \\
\hline \multicolumn{7}{|l|}{ Autismo } \\
\hline Inibição & $0,75^{* *}$ & $0,41 * *$ & 0,12 & $-0,04$ & 0,30 & 0,09 \\
\hline Impulsividade & $0,72 * *$ & $0,80 * *$ & 0,16 & 0,30 & 0,08 & 0,31 \\
\hline Emotiv. Neg. & $0,42 *$ & $0,73 * *$ & 0,15 & $0,57^{* *}$ & $-0,06$ & 0,32 \\
\hline Nível Atividade & $0,66^{* *}$ & $0,57 * *$ & 0,22 & 0,11 & 0,07 & 0,05 \\
\hline Falta Persist. & $0,57 * *$ & $0,76^{* *}$ & 0,32 & $0,40^{*}$ & $0,40^{*}$ & $0,52 * *$ \\
\hline Média $^{\mathrm{a}}$ & $0,64 * *$ & $0,68 * *$ & 0,20 & 0,29 & 0,16 & 0,27 \\
\hline \multicolumn{7}{|l|}{ Prematuros } \\
\hline Inibição & $0,83 * *$ & & $0,42 * *$ & $0,46^{*}$ & & \\
\hline Impulsividade & $0,58 * *$ & & 0,23 & $0,60 * *$ & & \\
\hline Emotiv.Neg. & $0,62 * *$ & & 0,32 & $0,46^{*}$ & & \\
\hline Nível Atividade & 0,26 & & $-0,16$ & 0,38 & & \\
\hline Falta Persist. & $0,54 * *$ & & 0,38 & 0,37 & & \\
\hline Média $^{a}$ & $0,60 * *$ & & 0,32 & 0,46 & & \\
\hline
\end{tabular}


Seabra-Santos, M. J. \& Almeida, M. S. (2014). Falamos da Mesma Criança? Concordância Mãe - Pai - Professores na Avaliação do Temperamento de Crianças Portuguesas.

Des. Normal

$\begin{array}{llcc}\text { Inibição } & 0,77^{* *} & 0,26 & 0,17 \\ \text { Impulsividade } & 0,69^{* *} & 0,31 & 0,18 \\ \text { Emotiv. Neg. } & 0,67^{* *} & 0,35^{*} & 0,32 \\ \text { Nível Atividade } & 0,59^{* *} & 0,10 & 0,01 \\ \text { Falta Persist. } & 0,63^{* *} & 0,30 & 0,23 \\ & 0,68^{* *} & 0,26 & 0,19\end{array}$

Nota . Prof.Reg. = Professor do Ensino Regular; Prof.Ap. = Professor dos Apoios Educativos; Emotiv. Neg. = Emotividade Negativa; Falta Persist. $=$ Falta de Persistência; Des. Normal $=$ Desenvolvimento Normal.

${ }^{a}$ As médias das correlações foram calculadas usando a transformação em $z$ de Fisher.

$* p<0,05 ; * *<0,01$.

A análise da Tabela 2 no que diz respeito à amostra total permite-nos verificar que: (a) o grau de concordância entre as avaliações dos informadores que observam a criança no mesmo contexto é elevado $(0,63$ e 0,73 , respetivamente entre mãe e pai, e entre os professores do ensino regular e dos apoios educativos) e muito superior ao verificado entre as avaliações de adultos que observam a criança em contextos diferentes (correlações na ordem de 0,20 a 0,30 entre as avaliações feitas por um dos progenitores - mãe ou pai - e as efetuadas por um dos professores); (b) o grau de concordância entre as avaliações feitas pelos dois professores é superior $(0,73)$ ao registado entre as avaliações de mãe e pai $(0,63)$; (c) os valores de concordância entre as avaliações de qualquer um dos progenitores e os professores do ensino regular são significativos e ligeiramente superiores aos valores registados entre os progenitores e os professores dos apoios educativos (respetivamente iguais a 0,24 e 0,29 , contra 0,22 e 0,20 ); (d) em geral verificam-se níveis mais elevados de concordância quanto à dimensão Impulsividade do que quanto à Inibição, com exceção da concordância entre os progenitores, que é superior para a dimensão Inibição; de entre as subdimensões da Impulsividade, a Falta de Persistência é aquela em que se regista um maior grau de concordância entre informadores.

O mesmo tipo de análise foi efetuado para cada uma das quatro subamostras separadamente (Tabela 2). Em geral, os resultados observados são idênticos aos obtidos para a amostra total. Relativamente à magnitude dos coeficientes de correlação encontrados em função da dimensão em avaliação, verificamos, também para as diversas subamostras, que os valores de concordância tendem a ser superiores para a dimensão Impulsividade que para a Inibição (ainda que haja flutuações pontuais), exceto no que diz respeito às avaliações feitas pelos progenitores, informadores que estão sistematicamente mais de acordo em relação à avaliação que fazem da Inibição do que da Impulsividade dos seus filhos. Por outro lado, no que respeita às subdimensões da Impulsividade, os resultados são variáveis entre as diversas subamostras, verificando-se uma tendência para valores de concordância mais elevados na subdimensão Falta de Persistência nas amostras com Síndrome de Down e com Autismo, enquanto se observam valores de concor- dância superiores na subdimensão Emotividade Negativa nas subamostras de crianças Nascidas Prematuramente e com Desenvolvimento Normal.

Quando comparamos as médias das pontuações atribuídas pelos vários informadores, para a amostra total e para cada uma das subamostras, verificamos que na maioria das dimensões e subdimensões não se observam diferenças significativas entre aquelas avaliações (Tabela 3). As únicas exceções verificam-se na dimensão Inibição, quer na amostra total, $F(2,387)=8,57, p<0,01$, quer na subamostra com Síndrome de Down, $F(3,127)=23,77$, $p<0,01$. Nestes casos os professores avaliam as crianças como mais inibidas do que os seus pais.

\section{Discussão}

Através deste estudo pretendeu-se analisar o grau de concordância entre diferentes informadores, designadamente, as mães, os pais, professores do ensino regular e professores dos apoios educativos (quando aplicável) na avaliação do temperamento de crianças em idade pré-escolar e início da idade escolar. Em síntese, os resultados vão no mesmo sentido dos apontados pela escassa literatura disponível (Achenbach et al., 1987; Martin \& Bridger, 1999; Martins, 2010; Muris \& Meesters, 2009), mostrando que as correlações entre as avaliações feitas a partir de informadores que observam a criança no mesmo contexto são significativas, elevadas e superiores às obtidas entre as avaliações de adultos que observam a criança em contextos diferentes, as quais traduzem níveis baixos de associação. Estes resultados têm implicações práticas importantes, salientando a utilidade de obter dados a partir de informadores presentes em contextos diversos (casa e escola), na medida em que estes representam contributos diferenciados para a avaliação. Por outro lado, tendo em conta a "economia" da avaliação em termos de tempo e recursos, poderá ser bastante redundante ter os mesmos questionários preenchidos pelo pai e pela mãe (Duhig et al., 2000), ou por mais que um professor. Saliente-se, no entanto, que um adulto que passa mais tempo com a criança está em melhores condições para fornecer um retrato objetivo das suas características. Neste sentido, as 
Psicologia: Reflexão e Crítica, 27(1), 10-20.

Tabela 3

Comparação entre as Médias das Pontuações Atribuidas pelos Vários Informadores em Resultados T (ANOVA)

\begin{tabular}{|c|c|c|c|c|c|}
\hline Amostras & $\begin{array}{c}\text { Mãe } \\
\text { média }(D P)\end{array}$ & $\begin{array}{c}\text { Pai } \\
\text { média }(D P)\end{array}$ & $\begin{array}{l}\text { Prof.Reg. } \\
\text { média }(D P)\end{array}$ & $\begin{array}{c}\text { Prof.Ap. } \\
\text { média }(D P)\end{array}$ & $F$ \\
\hline Total & $(n=138)$ & $(n=126)$ & $(n=126)$ & $(n=61)^{\mathrm{a}}$ & \\
\hline Inibição & $48,06(10,21)$ & $48,83(9,63)$ & $52,75(9,40)$ & & $8,57^{* *} \quad \mathrm{M}, \mathrm{P}<\mathrm{PR}$ \\
\hline Impulsividade & $53,38(9,95)$ & $53,67(9,62)$ & $52,42(8,96)$ & & 0,60 \\
\hline Emotiv. Neg. & $51,09(9,65)$ & $52,17(9,44)$ & $50,26(8,90)$ & & 1,33 \\
\hline Nível Atividade & $49,97(9,47)$ & $50,89(9,96)$ & $50,04(10,42)$ & & 0,34 \\
\hline Falta Persist. & $56,20(10,61)$ & $55,62(11,42)$ & $55,01(9,85)$ & & 0,42 \\
\hline Síndrome de Down & $(n=36)$ & $(n=32)$ & $(n=32)$ & $(n=31)$ & \\
\hline Inibição & $44,36(10,28)$ & $44,91(10,24)$ & $58,31(7,98)$ & $58,84(9,00)$ & $23,77^{* *} \mathrm{M}, \mathrm{P}<\mathrm{PR} . \mathrm{PA}$ \\
\hline Impulsividade & $51,78(10,18)$ & $53,06(10,36)$ & $52,56(7,59)$ & $53,55(8,08)$ & 0,23 \\
\hline Emotiv. Neg. & $49,42(11,14)$ & $51,81(9,82)$ & $47,22(6,53)$ & $48,68(8,75)$ & 1,37 \\
\hline Nível Atividade & $48,25(10,00)$ & $47,78(10,85)$ & $48,22(10,67)$ & $48,55(9,93)$ & 0,03 \\
\hline Falta Persist. & $58,14(11,89)$ & $58,56(10,50)$ & $59,41(8,77)$ & $60,06(8,66)$ & 0,24 \\
\hline Autismo & $(n=33)$ & $(n=29)$ & $(n=30)$ & $(n=30)$ & \\
\hline Inibição & $48,21(11,60)$ & $49,83(11,60)$ & $53,97(8,57)$ & $52,70(11,02)$ & 2,15 \\
\hline Impulsividade & $56,97(9,76)$ & $58,00(8,27)$ & $56,73(7,90)$ & $57,83(7,96)$ & 0,16 \\
\hline Emotiv.Neg. & $53,76(9,40)$ & $54,45(9,84)$ & $56,73(9,03)$ & $55,83(8,82)$ & 0,65 \\
\hline Nível Atividade & $51,70(9,40)$ & $53,38(8,39)$ & $52,10(9,07)$ & $54,60(9,62)$ & 0,64 \\
\hline Falta Persist. & $60,24(11,69)$ & $62,10(10,60)$ & $57,07(8,98)$ & $59,27(8,98)$ & 1,26 \\
\hline Prematuros & $(n=33)$ & $(n=30)$ & $(n=28)$ & $(n=5)$ & \\
\hline Inibição & $48,27(7,80)$ & $49,73(9,93)$ & $49,82(9,87)$ & - & 0,64 \\
\hline Impulsividade & $54,47(10,70)$ & $52,37(10,08)$ & $51,39(8,95)$ & - & 0,51 \\
\hline Emotiv. Neg. & $51,30(9,41)$ & $51,17(9,56)$ & $48,39(7,91)$ & - & 0,67 \\
\hline Nível Atividade & $51,66(9,59)$ & $51,20(10,39)$ & $49,07(12,19)$ & - & 0,32 \\
\hline Falta Persist. & $53,69(8,09)$ & $51,67(11,86)$ & $54,64(9,16)$ & - & 0,47 \\
\hline Des.Normal & $(n=36)$ & $(n=35)$ & $(n=36)$ & $(n=0)$ & \\
\hline Inibição & $51,42(9,90)$ & $50,83(9,28)$ & $49,08(8,54)$ & - & 0,62 \\
\hline Impulsividade & $50,72(8,33)$ & $51,77(8,82)$ & $49,50(9,85)$ & - & 0,56 \\
\hline Emotiv. Neg. & $50,14(8,25)$ & $51,49(8,72)$ & 49,03 $(8,99)$ & - & 0,72 \\
\hline Nível Atividade & $48,61(8,70)$ & $51,40(9,62)$ & $50,69(9,81)$ & - & 0,85 \\
\hline Falta Persist. & $52,81(8,75)$ & $50,94(9,23)$ & $49,67(9,81)$ & - & 1,04 \\
\hline
\end{tabular}

Nota . Prof.Reg./PR = Professor do Ensino Regular; Prof.Ap./PA = Professor dos Apoios Educativos; Emotiv. Neg. = Emotividade Negativa; Falta Persist. $=$ Falta de Persistência; Des.Normal $=$ Desenvolvimento Normal

${ }^{a}$ Os professores dos apoios educativos não foram considerados nas análises relativas à amostra total dado terem classificado somente crianças com necessidades educativas especiais e não a totalidade da amostra, o que poderia enviesar as conclusões dado estarem a ser comparadas médias de resultados que podem ser diferentes atendendo às diferentes características das subamostras em estudo. $* * p<0,01$. 
Seabra-Santos, M. J. \& Almeida, M. S. (2014). Falamos da Mesma Criança? Concordância Mãe - Pai - Professores na Avaliação do Temperamento de Crianças Portuguesas.

mães são, em geral, avaliadores mais fidedignos que os pais (Bishop et al., 2003), comprovando-se o mesmo em relação a professores com mais anos de experiência, por comparação com outros que possuam menos experiência (Anthony et al., 2005).

No presente estudo, o facto de os questionários respondidos pelos pais e pelos professores constituírem formas da TABC-R que são paralelas mas não exatamente iguais poderá, em parte, explicar a magnitude inferior dos coeficientes de correlação obtidos entre informadores que preencheram formas diferentes do inventário. Mais provavelmente, esta discrepância coloca em destaque a variabilidade do comportamento da criança em função do contexto, amplamente referida na literatura (Keith \& Campbell, 2004) e comprovada a partir de outros estudos empíricos (Achenbach et al., 1987; Martin \& Bridger, 1999; Merrell, 2002).

Na investigação sobre temperamento o foco da avaliação são características que se espera sejam relativamente estáveis entre contextos e ao longo do tempo (Seifer, 2003). Porém, tal não significa que qualquer traço particular se revele igualmente bem em todos os contextos, dado que cada meio possui características específicas, com diferente potencial para desencadear uma resposta (Rothbart \& Bates, 2006), nomeadamente devido à presença de diferentes pessoas com histórias prévias de interação com a criança (Martin, 2010). A este propósito são de enfatizar as especificidades do ambiente de jardim-de-infância, mais estruturado e envolvendo um maior número de contactos sociais que o ambiente de casa. Por conseguinte, quando avaliam o comportamento da criança os professores disporão não apenas de uma base normativa mais sólida como, igualmente, de indicadores comportamentais mais precisos onde ancorar as suas avaliações. Tal pode justificar a superioridade da correlação encontrada entre os registos dos professores, em comparação com a observada entre os dos pais. Por outro lado, os valores de concordância entre as avaliações de qualquer um dos progenitores e as dos professores do ensino regular são significativos e superiores aos valores registados entre os progenitores e os professores dos apoios educativos. Tal resultado poderá estar relacionado com um menor tempo de contacto com as crianças por parte dos professores dos apoios, o que se pode refletir num menor nível de conhecimento e, em consequência, diminuir o grau de concordância entre estes e outros informadores (Anthony et al., 2005).

No que diz respeito às diferentes dimensões avaliadas pela TABC-R, em geral verificam-se níveis mais elevados de concordância na dimensão Impulsividade do que na Inibição, refletindo um menor insight dos informadores relativamente a características que têm uma tradução comportamental menos evidente. Assim, a Impulsividade é avaliada através de comportamentos observáveis e mais fáceis de detetar (por exemplo, "Quando fala com alguém, dá pulinhos de excitação") do que os relativos à característica Inibição (por exemplo, "Mostra-se tímido quando conhece novas crianças"), o que se reflete em níveis de concordância entre informadores mais elevados no primeiro caso do que no segundo (Berg-Nielsen et al., 2012; Muris \& Meesters, 2009)". A análise da concordância entre informadores em cada uma das subamostras reflete as mesmas tendências gerais verificadas para a amostra total. Por conseguinte, variáveis como a idade da criança no momento do diagnóstico - diferente nos casos de Síndrome de Down e Prematuridade (diagnóstico feito à nascença) e nos casos de Autismo (idade variável) -, ou o conhecimento que os professores têm em relação a esses diagnósticos - presente nos casos de Síndrome de Down e Autismo mas só raramente nos de Prematuridade - embora podendo criar expetativas diferentes nos adultos em relação a estes grupos de crianças, não parecem ter impacto sobre a concordância entre eles na avaliação do temperamento daquelas. Porém, no que diz respeito à concordância registada para as subdimensões da Impulsividade existem flutuações entre as amostras, refletindo uma tendência para valores de concordância mais elevados nas subdimensões relativas a características mais salientes ou importantes para o ajustamento das crianças em causa (Field \& Greenberg, 1982). Assim, a concordância tende a ser mais acentuada em relação à Falta de Persistência para as subamostras com Síndrome de Down e com Autismo, enquanto para as crianças Nascidas Prematuramente ou com Desenvolvimento Normal é a subdimensão Emotividade Negativa (gritar e lutar, amuar ou ser difícil de consolar) que alcança maiores níveis de concordância (Martin \& Bridger, 1999).

A única diferença estatisticamente significativa entre as médias das pontuações atribuídas pelos vários informadores, verifica-se na dimensão Inibição, quer na amostra total, quer nas subamostras com Síndrome de Down e com Autismo (embora neste último caso a diferenças não tenha significado estatístico). O facto de, nestes casos, os professores registarem um maior grau de inibição nas crianças do que os seus pais, poderá indicar que crianças com atraso de desenvolvimento manifestam comportamentos relacionados com inibição (tais como ser tímido com pessoas que não conhece ou levar muito tempo a ficar à vontade numa nova situação) mais na escola do que em casa. Por outro lado, se a convergência entre informadores é um indicador (embora não suficiente) da fidedignidade de uma avaliação, os nossos dados apontam os pais como informadores mais fiáveis do que os professores nas apreciações da inibição comportamental das crianças, dado o elevado nível de concordância registado entre mãe e pai quanto àquela característica (Bishop et al., 2003).

O presente estudo comporta alguns aspetos positivos, ao mesmo tempo que algumas limitações. Os principais contributos referem-se ao facto de se ter recorrido a vários informadores (três ou quatro conforme os casos) em dois contextos diferentes (casa e escola), incluindo os professores dos apoios educativos e os pais-homens, sendo estes últimos informadores com frequência negligenciados na investigação sobre crianças (Duhig et al., 2000). Por outro lado, é analisado não só o grau de concordância mas, igualmente, as divergências entre os informadores (diferenças 
entre médias) e em quatro subamostras com características distintas. Como principais limitações regista-se o recurso a normas americanas para derivação dos resultados $T$, na ausência de normas para a população portuguesa. Neste contexto, e apesar das boas qualidades psicométricas atestadas pelos estudos até à data realizados com a TABC-R em Portugal (Almeida \& Seabra-Santos, 2010; Martins, 2010), salienta-se a necessidade de uma verdadeira aferição nacional, assente numa amostra representativa da população pré-escolar portuguesa. A heterogeneidade da amostra usada no presente estudo e o reduzido número de sujeitos em cada uma das subamostras limita também o alcance das conclusões. Por outro lado, poderá constituir um aspeto negativo o facto de a coleta de dados ter seguido percursos diferentes no caso das amostras clínicas (em que o primeiro contacto foi feito através dos pais) e da amostra com desenvolvimento normal (abordagem iniciada através dos professores). Também não foi analisada a concordância entre avaliadores em função de diferentes níveis de formação ou do grau de conhecimento das crianças por parte dos professores, embora estas variáveis sejam reconhecidas como relevantes (Anthony et al., 2005; Mashburn, Hamre, Downer, \& Pianta, 2006).

Os resultados alcançados suscitam algumas reflexões quanto à avaliação do temperamento. Em primeiro lugar quanto ao uso de inventários respondidos por terceiros. Apesar de a utilização deste tipo de instrumentos levantar vários problemas quanto à sua validade (Carey \& McDevitt, 2012; Kagan \& Fox, 2006), o recurso a questionários e ao testemunho de terceiros na avaliação do temperamento pré-escolar é incontornável (Martin, 2011; Rothbart \& Bates, 2006), sendo reconhecido o seu contributo no rastreio de crianças em risco de desenvolver diversos problemas devido aos seus traços temperamentais extremos (Bishop et al., 2003; McDevitt, 2011).

Um segundo conjunto de reflexões tem a ver com as características dos próprios questionários. Do ponto de vista da construção de instrumentos, a discordância apela para a necessidade de derivar normas separadas, nomeadamente para pais e para professores. Por outro lado, o uso de intervalos de frequência mais claros na escala de resposta dos inventários (por exemplo, "1 a 2 vezes por semana", em vez de "quase nunca") poderia conferir um maior grau de objetividade às classificações atribuídas pelos informadores e aumentar o grau de concordância entre eles. Podemos, igualmente, colocar a hipótese de que itens relativos a situações concretas e a comportamentos diretamente observáveis (e.g, "O meu filho remexe-se constantemente quando está sentado à mesa") possam suscitar um maior grau de concordância do que itens mais abstratos, que envolvam um maior grau de inferência e generalização (e.g., "O meu filho/a tem bom feitio"). Neste sentido, os estudos relativos à concordância na avaliação do temperamento (leia-se, disposições comportamentais) poderiam chegar a índices mais baixos do que os obtidos para comportamentos mais diretamente observáveis. Os resultados do presente estudo não apontam para níveis de concordância inferiores na avaliação do temperamento da criança, em comparação com estudos que visam a concordância na avaliação do comportamento (cf. Achenbach et al., 1987). Porém, será útil que estudos futuros explorem esta questão com maior detalhe, nomeadamente comparando o grau de concordância obtido para itens que envolvem diferentes níveis de inferência. Neste contexto, a observação direta, conduzida por observadores treinados, poderia constituir uma alternativa de avaliação mais objetiva do que os inventários (Dougherty et al., 2011). Contudo, comporta também vários inconvenientes, nomeadamente relativos ao dispêndio de tempo e de recursos e à dificuldade de generalização a partir de uma situação laboratorial para contextos de vida real (Martin, 2010).

Uma terceira reflexão diz respeito à necessidade de explorar as causas das divergências entre os testemunhos, uma vez que estas poderão traduzir diferenças de perspetiva que podem ser úteis para apreciar os distintos aspetos de uma realidade socialmente complexa (Bates, 1994). Nos últimos anos tem sido consistentemente realçada a relevância da avaliação do temperamento da criança e da necessidade de ajustar as condições do meio em função dessas características (Carey \& McDevitt, 2012). Neste contexto, e dado que as respostas aos questionários envolvem a combinação de fatores objetivos e subjetivos (Bates, 1994), devem ser claramente discriminadas as causas para a divergência entre relatos: traduzem diferenças reais no comportamento da criança ou sobretudo divergências nas perceções dos informadores? A resposta a esta questão poderá conduzir a processos de intervenção distintos, nuns casos mais direcionados para as crianças, noutros para os adultos. Em muitas situações, a exploração das causas da discordância poderá pôr em destaque variações no modo de funcionar da criança de situação para situação e em função do parceiro na interação (Martin, 2010). Por conseguinte, as diferenças entre o comportamento da criança em casa e na escola, por exemplo, podem apontar para a necessidade de implementar intervenções usando técnicas distintas e com objetivos diversos (Achenbach et al., 1987; Klyce et al., 2011). Por outro lado, a observação de tais divergências poderá ajudar a compreender o modo como determinadas características presentes num ou noutro contexto contribuem para potenciar ou inibir certos comportamentos na criança. Neste sentido, é importante salientar o contributo positivo que a análise das discordâncias entre informadores pode oferecer, ao chamar a atenção para algumas características da criança que podem ser moldadas em função do tipo específico de estimulação que recebem do meio e para as condições que melhor favorecem a expressão mais positiva de tais características (Murray, Ruble, Willis, \& Molloy, 2009). Nesta mesma linha, estudos futuros poderão, igualmente, tentar compreender se os níveis de convergência entre adultos são ou não afetados por fatores de natureza cultural. Isto é, haverá culturas em que as práticas educativas adotadas nos contextos familiar e escolar se aproximam mais do que noutras? E poderá tal circunstância refletir-se em níveis 
Seabra-Santos, M. J. \& Almeida, M. S. (2014). Falamos da Mesma Criança? Concordância Mãe - Pai - Professores na Avaliação do Temperamento de Crianças Portuguesas.

diferentes de convergência, na avaliação do temperamento das crianças?

Concluindo, o recurso a uma abordagem multi-informadores na avaliação do temperamento afigura-se como um meio indispensável de aumentar a precisão da avaliação (Muris \& Meesters, 2009). Por conseguinte, a investigação na área do temperamento não deve focar-se na pessoa independentemente do seu contexto e na avaliação dos atributos temperamentais de uma forma isolada (Lerner \& Lerner, 1983), mas deve esforçar-se por compreender o modo como os diferentes filtros influenciam a nossa compreensão do fenómeno (Kagan \& Fox, 2006; Rothbart \& Bates, 2006; Seifer, 2003). A este respeito Kagan e Fox avançam, mesmo, a possibilidade de

os psicólogos poderem vir a substituir os construtos atuais, que descrevem as crianças e os seus meios (pais, irmãos e contextos escolares) separadamente, por construtos sintéticos e únicos, que representem um tipo temperamental particular, a desenvolver-se num conjunto particular de contextos (2006, p. 216).

A noção de bom ajustamento ("goodness of fit") introduzida por Chess e Thomas $(1989,1999)$ e tão cara aos teóricos do temperamento (Carey \& McDevitt, 2012) preconiza, precisamente, que pais, professores, técnicos e profissionais de saúde, em trabalho conjugado, assegurem que as características e necessidades de cada criança encontram nos seus diversos contextos as oportunidades, exigências e expectativas capazes de assegurar o desenvolvimento ótimo das suas potencialidades.

\section{Referências}

Achenbach, T. M., McConaughy, S. H., \& Howell, C. T. (1987). Child/adolescent behavioral and emotional problems: Implications of cross-informant correlations for situational specificity. Psychological Bulletin, 101, 213-232.

Almeida, M., \& Seabra-Santos, M. J. (2010). Bateria de Avaliação do Temperamento Infantil - Forma Revista. Psychologica, 53(3), 313-328.

Anthony, B. J., Anthony, L. G., Morrel, T. M., \& Acosta, M. (2005). Evidence for social and behavioral problems in lowincome, urban preschoolers: Effects of site, classroom, and teachers. Journal of Youth and Adolescence, 34(1), 31-39.

Bates, J. E. (1994). Parents as scientific observers of their children's development. In S. L. Friedman \& H. C. Haywood (Eds.), Developmental follow-up: Concepts, domains and methods (pp. 197-216). San Diego, CA: Academic Press.

Berg-Nielsen, T. S., Solheim, E., Belsky, J., \& Wichstrom, J. (2012). Preschoolers' psychosocial problems: In the eyes of the beholder? Adding teacher characteristics as determinants of discrepant parent-teacher reports. Child Psychiatry and Human Development, 43, 393-413. doi:10.1007/s10578011-0271-0

Bishop, G., Spence, S. H., \& McDonald, C. (2003). Can parents and teachers provide a reliable and valid report of behavioral inhibition? Child Development, 74(6), 1899-1917.

Carey, W. C., \& McDevitt, S. C. (2012). Child behavior assessment and management in primary care: Theory and Practice. Retrieved May 21, 2012, from http://www.b-di. com/CBAM.html
Chess, S., \& Thomas, A. (1989). Issues in clinical application of temperament. In G. A. Kohnstamm, J. E. Bates, \& M. K. Rothbart (Eds.), Temperament in childhood (pp. 377-386). New York: John Wiley \& Sons.

Chess, S., \& Thomas, A. (1999). Goodness of fit: Clinical applications from infancy through adult life. Philadelphia, PA: Brunner.

Dougherty, L. R., Bufferd, S. J., Carlson, G. A., Dyson, M., Olino, T. M., Durbin, C. E., \& Klein, D. N. (2011). Preschoolers' observed temperament and psychiatric disorders assessed with a parent diagnostic interview. Journal of Clinical Child and Adolescent Psychology, 40(2), 295-306. doi:10.1080/15 374416.2011.546046

Duhig, A. M., Renk, K., Epstein, M. K., \& Phares, V. (2000). Interparental agreement on internalizing, externalizing, and total behavior problems: A meta-analysis. Clinical Psychology: Science and Practice, 7(4), 435-453.

Field, T., \& Greenberg, R. (1982). Temperament ratings by parents and teachers of infants, toddlers, and preschool children. Child Development, 53, 160-163.

Forman, D. R., O'Hara, M. W., Larsen K., Coy, K. C., Gorman, L. L., \& Stuart, S. (2003). Infant emotionality: Observational methods and the validity of maternal reports. Infancy, 4, 541-565.

Goldberg, W. A. (1990). Marital quality, parental personality, and spousal agreement about perceptions and expectations for children. Merrill-Palmer Quarterly, 36(4), 531-556.

Goldsmith, H. H., Buss, A. H., Plomin, R., Rothbart, M. K., Thomas, A., Chess, S., ...McCall, R. B. (1987). Roundtable: What is temperament? Four approaches. Child Development, 58, 505-529.

Hayden, E. P., Durbin, C. E., Klein, D. N., \& Olino, T. M. (2010). Maternal personality influences the relationship between maternal reports and laboratory measures of child temperament. Journal of Personality Assessment, 92(6), 586593. doi:10.1080/00223891.2010.513308

Kagan, J., \& Fox, N. A. (2006). Biology, culture and temperamental biases. In W. Damon \& R. M. Lerner (Eds.-in-chief) \& N. Eisenberg (Vol. Ed.), Handbook of child development: Vol. 3. Social, emotional and personality development $\left(6^{\text {th }}\right.$ ed., pp. 167-225). New York: John Wiley \& Sons.

Keith, L. K., \& Campbell, J. M. (2004). Assessment of social and emotional development in preschool children. In B. A. Bracken (Ed.), The psychoeducational assessment of preschool children ( $3^{\text {rd }}$ ed., pp. 364-382). Mahwah, NJ: Lawrence Erlbaum.

Keogh, B. K., \& Bernheimer, L. P. (1998). Concordance between mothers' and teachers' perceptions of behaviour problems of children with developmental delays. Journal of Emotional and Behavioral Disorders, 6(1), 33-41.

Klyce, D., Conger, A. J., Conger, J. C., \& Dumas, J. E. (2011). Measuring competence and dysfunction in preschool children: Source agreement and component structure. Journal of Child and Family Studies, 20, 503-510. doi:10.1007/s10826-0109417-0

Kristal, J. (2005). The temperament perspective: Working with children's behavioral styles. New York: Paul Brookes.

Kroes, G., Veerman, J. W., \& De Bruyn, E. E. J. (2005). The impact of the Big Five personality traits on reports of child behavior problems by different informants. Journal of Abnormal Child Psychology, 33, 231-240.

Lerner, J. V., \& Lerner, R. M. (1983). Temperament and adaptation across life: Theoretical and empirical issues. In P. B. 
Baltes \& O. G. Brim Jr. (Eds.), Life-span development and behavior (Vol. 5, pp. 197-231). New York: Academic Press.

Macedo, A., Marques, M., Bos, S., Maia, B. R., Pereira, T., Soares, M. J., ...Azevedo, M. H. (2011). Mother's personality and infant temperament. Infant Behavior and Development, 34(4), 552-568. doi:10.1016/j.infbeh.2011.06.009

Major, S. O. (2011). Avaliação de aptidões sociais e problemas de comportamento em idade pré-escolar: Retrato das crianças portuguesas (Tese de doutorado, Faculdade de Psicologia e de Ciências da Educação, Universidade de Coimbra, Coimbra, Portugal). Recuperado em https://estudogeral.sib.uc.pt/ bitstream/10316/17774/5/Tese_Sofia\%20Major.pdf

Martin, R. P. (2010). Thoughts on temperament research. Newsletter of the Temperament Consortium, 1(2), 4-5. Retrieved May 7, 2012, from http:/www.b-di.com/pdf/ TCNewsSpring10.pdf

Martin, R. P. (2011). Thoughts on temperament research, from the editor. Newsletter of the Temperament Consortium, 2(1), 3. Retrieved May 7, 2012, from http://www.b-di.com/pdf/ TCNewsSpring11.pdf

Martin, R. P., \& Bridger, R. C. (1999). Temperament Assessment Battery for Children-Revised. A tool for the assessment of temperamental traits and types of young children: Manual. Athens, GA: School Psychology Clinic Publishers.

Martins, S. P. (2010). O temperamento das crianças e os estilos e sentido de competência parentais (Dissertação de mestrado, Faculdade de Psicologia e de Ciências da Educação, Universidade de Coimbra, Coimbra, Portugal).

Mashburn, A. J., Hamre, B. K., Downer, J. T., \& Pianta, R. C. (2006). Teacher and classroom characteristics associated with teachers' ratings of prekindergartners' relationships and behaviors. Journal of Psychoeducational Assessment, 24(4), 367-380. doi:10.1177/07342829062905

McClowry, S. G., Halverson, C. F., \& Sanson, A. (2003). A reexamination of the validity and reliability of the School-Age Temperament Inventory. Nursing Research, 52(3), 176-182.

McDevitt, S. C. (2011). Temperament, adjustment \& psychopathology: A proposed taxonomy of fit with implications for clinical assessment and management. Newsletter of the Temperament Consortium, 2(1), 4. Retrieved May 7, 2012, from http://www.b-di.com/pdf/TCNewsSpring11.pdf

Merrell, K. W. (2002). Preschool and Kindergarten Behavior Scales - Second Edition. Austin, TX: PRO-ED.

Miner, J. L., \& Clarke-Stewart, K. A. (2008). Trajectories of externalizing behavior from age 2 to age 9: Relations with gender, temperament, ethnicity, parenting, and rater. Developmental Psychology, 44(3), 771-786. doi:10.1037/00121649.44.3.771

Muris, P., \& Meesters, C. (2009). Reactive and regulative temperament in youths: Psychometric evaluation of the Early Adolescent Temperament Questionnaire-Revised. Journal of Psychopathology and Behavioral Assessment, 31, 7-19.

Murray, D. S., Ruble, L. A., Willis, H., \& Molloy, C. A. (2009). Parent and teacher report of social skills in children with autism spectrum disorders. Language, Speech, and Hearing Services in Schools, 40, 109-115.

Rochette, S. E. (2008). The relationship between parent and teacher ratings on the Adjustment Scales for Children and Adolescents (Doctoral dissertation). Retrieved from ProQuest Dissertations \& Theses, UMI Microform No 3336116.
Rothbart, M. K., \& Bates, J. E. (2006). Temperament. In W. Damon, R. M. Lerner (Eds.-in-chief) \& N. Eisenberg (Vol. Ed.), Handbook of child development: Vol. 3. Social, emotional and personality development ( $6^{\text {th }}$ ed., pp. 99-166). New York: John Wiley \& Sons.

Seabra-Santos, M. J. (2000). Avaliação psicológica em idade pré-escolar: O caso da avaliação da inteligência. Psychologica, $25,143-162$.

Seifer, R. (2003). Twin studies, biases of parents, and biases of researchers. Infant Behavior \& Development, 26, 115-117.

Weisz, J. R., Sigman, M., Weiss, B., \& Mosk, J. (1993). Parent reports of behavioral and emotional problems among children in Kenya, Thailand, and the United States. Child Development, 64, 98-109. 